\title{
STRUCTURAL UNCERTAINTY DETERMINATION BY STATISTICAL. ANALYSIS
}

ANDRÉ HAAS, PIERRE THORE and JEAN ARNAUD

Elf Aquitaine Production, Division Recherches Géologiques et Géophysiques-CSTJF, avenue Larribau, 64018 Pau Cedex, France

\section{Introduction}

Numlerous methods can be used to determine a depth macro-model from seismic data, generally by calculation of interval velocities, their interpolation and migration of seismic times with these velocities.

The accuracy of the calculated depth is important and depends mainly on the uncertainties of the velocities used for the migration.

In this paper, we present two methods to calculate this depth uncertainty :

a simplified method used on 3D which gives the opportunity to test assumptions on the velocity field,

- a statistical method, at present 2D only, which allows a finer evaluation of the depth uncertainty.

\section{Simplified method}

Given a depth model in a constant velocity medium a variation $\Delta \mathrm{V}$ of this velocity causes a variation of the depth point location which is not vertical but circular and depends on the depth and the local dip of the horizon (see an anticline model in Fig. 1).

For a $45^{\circ}$ dip, the variation has no vertical component.

In a $V_{0}+k z$ medium, an analytic calculation can be performed involving the local dip, its azimuth and the migration offset.

Fig. 2 shows an application on a synthetic "salt dome" model with overhang in a medium of interval velocity $1700+0,7 \mathrm{Z}$ and $\Delta \mathrm{V} / \mathrm{V} 2 \%$. The figure shows a $\mathrm{GOCAD}^{\mathrm{m}}$ display of the original depth model, the directions of uncertainty and around the model the interpolated uncertainties.

This method was successfully applied to calibrate a depth surface to well data along the main directions of uncerainties.

\section{Layer stripping method}

When velocity contrasts and dip unconformities are prcscui (layer stripping model), we use a statistical method which enables us to calculate the probabilities of location of the studied horizon at a given depth.
The method is currently used in 2D but will be implemented in 3D in a few months.

Given the best layer stripping model derived from a velocity interpretation, and given uncertainties on each interval velocity, we can calculate randomly interval velocities and numerous equiprobable new models.

500 depth points are drawn randomly from Gaussian distribution. This events a cloud of points for each selected CDP location along the deepest horizon.

The intermediate horizons are automatically interpolated with spline functions.

Fig. 3 shows the principle of this method, and Fig. 4 its application to a real case study.

The software runtime is between 20 minutes and 1 hour for 500 random drawings on a SUN SPARC 2 workstation depending on the number of calculated locations. As a result, it is realistic to make several velocity and uncertainty hypotheses.

In the real case illustrated on Fig. 4, we were able to better calibrate the well to seismic data for the depth and the dip of the horizon.

It is also possible to give an uncertainty in the stacking velocities and after generating statistical realizations apply the DIX formula to take into account the velocity correlation between two consecutive levels.

More generally, it is possible to take into account a vertical correlation between the velocities.

\section{Conclusion}

The two methods presented in this paper allow the evaluation of the depth uncertainty on a horizon as a function of the velocity uncertainties of the upper horizons.

Using these methods, different velocity and uncerainty hypotheses can be easily tested and well calibration can be performed along the main direction of uncertainty. 

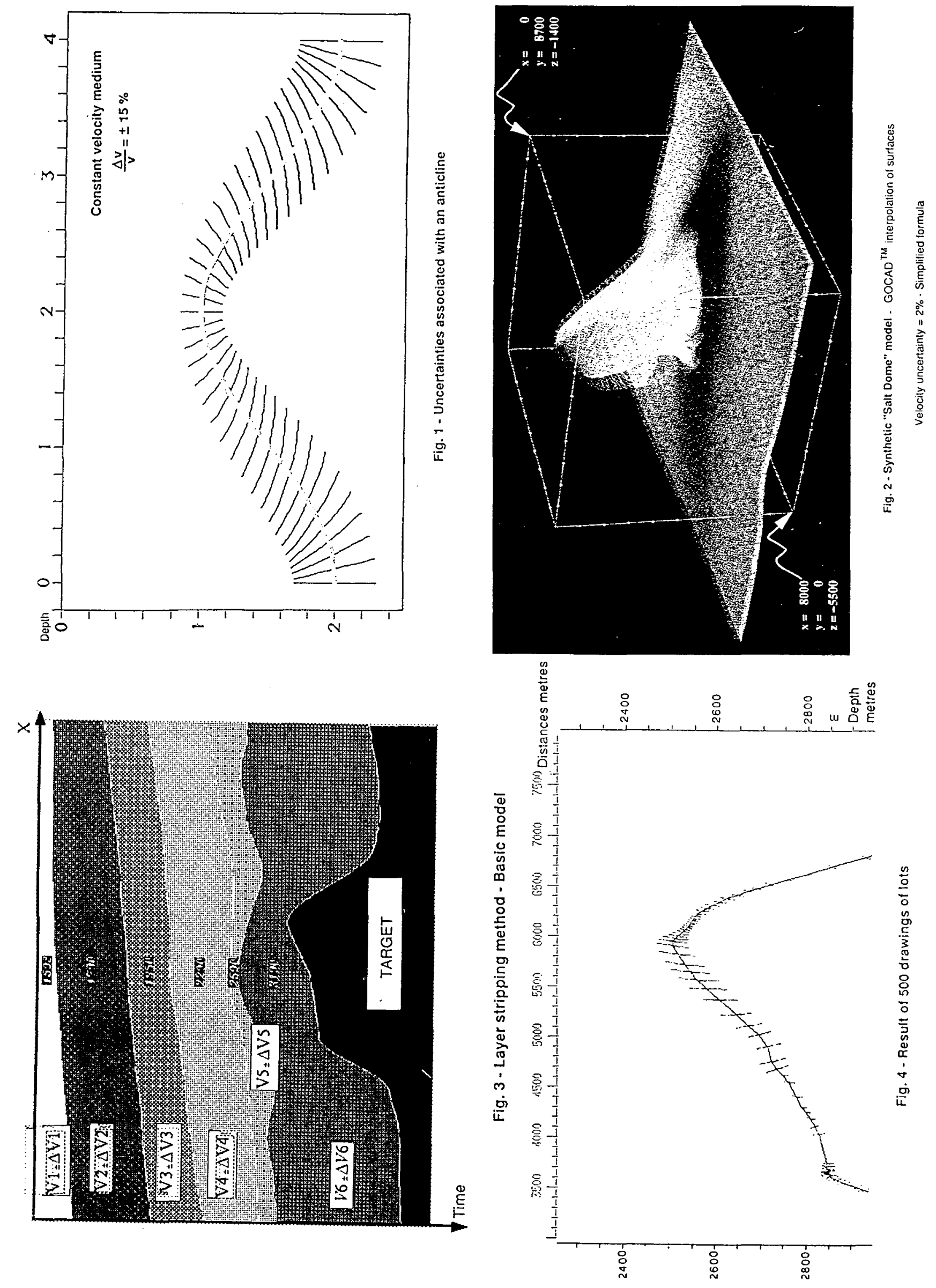